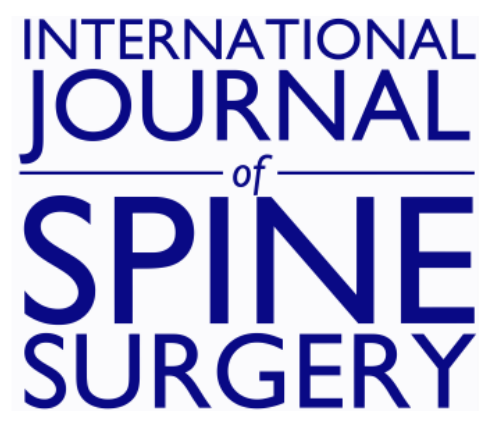

\title{
Trends in Pain Medication Prescriptions and Satisfaction Scores in Spine Surgery Patients at a Single Institution
}

ERIK WANG, DENNIS VASQUEZ-MONTES, DEEPTEE JAIN, LORRAINE H. HUTZLER, JOSEPH A. BOSCO, THEMISTOCLES S. PROTOPSALTIS, AARON J. BUCKLAND and CHARLA R. FISCHER

Int J Spine Surg 2020, 14 (6) 1023-1030

doi: https://doi.org/10.14444/7153

http://ijssurgery.com/content/14/6/1023

This information is current as of April 26, 2023.

Email Alerts Receive free email-alerts when new articles cite this article. Sign up at: http://ijssurgery.com/alerts 


\title{
Trends in Pain Medication Prescriptions and Satisfaction Scores in Spine Surgery Patients at a Single Institution
}

ERIK WANG, BA, DENNIS VASQUEZ-MONTES, MS, DEEPTEE JAIN, MD, LORRAINE H. HUTZLER, MPA, JOSEPH A. BOSCO, MD, THEMISTOCLES S. PROTOPSALTIS, MD, AARON J. BUCKLAND, MBBS, FRACS, CHARLA R. FISCHER, MD

Department of Orthopedics, NYU Langone Orthopedic Hospital, New York University, New York, NY

\begin{abstract}
Background: As the opioid crisis has gained national attention, there have been increasing efforts to decrease opioid usage. Simultaneously, patient satisfaction has been a crucial metric in the American health care system and has been closely linked to effective pain management in surgical patients. The purpose of this study was to examine rates of pain medication prescription and concurrent patient satisfaction in spine surgery patients.

Methods: A total of 1729 patients undergoing spine surgery between June 25, 2017, and June 30, 2018, at a single institution by surgeons performing $\geq 20$ surgeries per quarter, with medication data during hospitalization available, were assessed. Patients were evaluated for nonopioid pain medication prescription rates and morphine milligram equivalents (MME) of opioids used during hospitalization. Of the total cohort, 198 patients were evaluated for Press Ganey Satisfaction Survey responses. A $\chi^{2}$ test of independence was used to compare percentages, and 1-way analysis of variance was used to compare means across quarters.

Results: The mean total MME per patient hospitalization was 574.46, with no difference between quarters. However, mean MME per day decreased over time $(P=.048)$, with highest mean 91.84 in Quarter 2 and lowest 77.50 in Quarter 4. Among all procedures, acetaminophen, nonsteroidal anti-inflammatory drugs, and steroid prescription rates increased, whereas benzodiazepine and $\gamma$-aminobutyric acid-analog prescriptions decreased. There were no significant differences between quarters for mean hospital ratings $(P=.521)$ nor for responses to questions from the Press Ganey Satisfaction Survey regarding how often staff talk about pain $(P=.164)$, how often staff talk about pain treatment $(P=$ $.595)$, or whether patients recommended the hospital $(P=.096)$. There were also no differences between quarters for responses in all other patient satisfaction questions ( $P$ value range, .359-.988).

Conclusions: Over the studied time period, opioid use decreased and nonopioid prescriptions increased during hospitalization, whereas satisfaction scores remained unchanged. These findings indicate an increasing effort in reducing opioid use among providers and suggest the ability to do so without affecting overall satisfaction rates.

Level of Evidence: 4.

Clinical Relevance: The opioid epidemic has highlighted the need to reduce opioid usage in orthopedic spine surgery. This study reviews the trends for inpatient management of post-op pain in orthopedic spine surgery patients in relation to patient satisfaction. There was a significant increase in non-opioid analgesic pain medications, and a reduction in opioids during the study period. During this time, patient satisfaction as measured by Press-Ganey surveys did not show a decrease. This demonstrates that treatment of post-operative pain in orthopedic spine surgery patients can be managed with less opioids, more multimodal analgesia, and patient satisfaction will not be affected.
\end{abstract}

Other \& Special Categories

Keywords: pain medication, opioids, spine surgery, patient satisfaction, Press Ganey

\section{INTRODUCTION}

Perioperative pain management presents a substantial challenge in spine surgery, because pain can be severe and lead to increased morbidity and complications if poorly controlled. Patients may suffer from delayed mobilization, extended hospital stay, slower functional recovery, development of chronic pain syndromes, and higher readmission rates if their pain is not effectively managed. ${ }^{1-7}$
Pugely et $\mathrm{al}^{7}$ assessed lumbar spine surgery patients for unplanned 30-day readmissions and found that pain-related reasons $(22.4 \%)$ were behind only wound-related complications $(38.6 \%)$ as the most frequent cause for readmission. Opioid analgesics have long been the first-line for postoperative pain management in major surgeries, including those involving the spine, with sales and distribution in the United States increasing nearly 4-fold between 
1997 and $2010 .^{8}$ However, opioid overuse has also been associated with significant adverse effects, including urinary retention, confusion, nausea and vomiting, constipation, respiratory depression, and even death. ${ }^{9,10}$

As the opioid crisis has gained national attention, there has been an increasing effort to decrease opioid usage without compromising effective pain control. Prescription opioid use has been shown to increase the risk of drug overdose by a factor of 6 and opioid dependence was estimated ${ }^{11,12}$ to be as high as $26 \%$ among patients receiving pain treatment in 2006. In 2008, opioids were implicated in 14800 deaths in the United States, a 3-fold increase over the course of a decade. ${ }^{13}$ Nonopioid therapies have been increasingly used as part of individualized, multimodal analgesia (MMA) regimens in an effort to improve pain control and minimize opioidrelated side effects. ${ }^{10,14,15}$ Studies $^{16,17}$ have reported on the capability of these MMA regimens to effectively reduce postoperative pain while also decreasing total opioid use. By relying on the synergistic action of multiple nonopioid medications, MMA regimens are able to manage pain with less of the unfavorable side effects associated with increased opioid usage.

Simultaneously, patient satisfaction is a crucial metric in the American health care system and has been closely linked to effective pain management in surgical patients. Patients who have less pain, better physical function, and a more positive impression of their hospital care after surgery have higher satisfaction scores. ${ }^{18-20}$ Given this connection and the increased rates of opioid use in recent years, some authors ${ }^{21}$ have cautioned clinicians about attempts to optimize patient satisfaction scores by prescribing more opioids. However, recent studies have also shown promise in the ability of MMA regimens to reduce opioid use without compromising satisfaction scores. Jirarattanaphochai et $\mathrm{al}^{22}$ found in 2008 that the addition of parecoxib versus a saline placebo after lumbar discectomy, decompression, or fusion procedures led to a $39 \%$ reduction in morphine use, along with reduced pain at rest and greater satisfaction. The purpose of this study was, therefore, to examine rates of pain medication prescription, both opioid and nonopioid, and concurrent patient satisfaction in spine surgery patients at our institution, where active efforts have been made to reduce opioid usage.

\section{MATERIALS AND METHODS}

\author{
Data Source and Collection
}

A retrospective cohort study between June 25, 2017, and June 30, 2018, was conducted for adult patients ( $>18$ years) undergoing any spine procedure at a single institution by spine surgeons performing at least 20 surgeries per quarter and whose data for medication during hospitalization were available. The year was split into quarters by date: Quarter 1 (Q1) spanning June 25, 2017September 30, 2017; Q2 spanning October 1, 2017-December 30, 2017; Q3 spanning December 31, 2017-March 31, 2018; and Q4 spanning April 1, 2018-June 30, 2018. Chart review was performed for demographic information (age and gender) and operative details (physician and procedure type), as well as pain medication usage. Procedure type was divided into groups for discectomy, fusion, laminectomy, and other.

\section{Pain Medication Use}

Patient records were assessed for nonopioid pain medication prescriptions during hospitalization as part of a multimodal analgesia regimen. Medications assessed included acetaminophen, nonsteroidal anti-inflammatory drugs (NSAIDs), steroids, benzodiazepines, and $\gamma$-aminobutyric acid (GABA) analogs. Patient records were also assessed for length of stay and opioid usage during hospitalization. Opioid medication usage was quantified in morphine milligram equivalents (MME) by per-day usage and also by usage for the total duration of hospitalization. The MME was calculated using conversion factors provided by the Centers for Medicare and Medicaid Services (https://www.cms. gov/Medicare/Prescription-Drug-Coverage/Pre scriptionDrugCovContra/Downloads/Opioid-Mor phine-EQ-Conversion-Factors-Aug-2017.pdf). Nonopioid pain medication prescription rates and opioid medication usage were assessed by quarter over the entire patient cohort and also subanalyzed by surgeon and procedure type.

\section{Press Ganey Satisfaction Survey Response}

Press Ganey Satisfaction Survey responses were assessed among the patients who voluntarily completed the survey to evaluate patient satisfaction. These questions had answer choices: never, sometimes, usually, and always. These answer choices were assigned values $0,1,2$, and 3 , respectively for 
Table 1. Opioid medication usage during hospitalization per patient by quarter. ${ }^{\text {a }}$

\begin{tabular}{|c|c|c|c|c|c|}
\hline Parameter & Q1 (n= 427) & Q2 $(n=439)$ & Q3 $(n=453)$ & $Q 4(n=440)$ & $P$ Value \\
\hline \multicolumn{6}{|l|}{ Total MME } \\
\hline Mean & 615.06 & 607.89 & 572.17 & 504.05 & .116 \\
\hline $95 \% \mathrm{CI}$ & $530.71-699.47$ & $525.94-689.83$ & $517.87-626.47$ & $445.02-563.09$ & \\
\hline \multicolumn{6}{|l|}{$\mathrm{MME} / \mathrm{d}$} \\
\hline Mean & 86.92 & 91.84 & 84.98 & 77.50 & .048 \\
\hline $95 \% \mathrm{CI}$ & $79.87-93.97$ & $83.29-100.40$ & $78.59-91.37$ & $70.88-84.12$ & \\
\hline Length of stay, d & 6.84 & 6.68 & 7.00 & 6.53 & .087 \\
\hline
\end{tabular}

Abbreviations: MME, morphine milligram equivalents; Q, quarter.

${ }^{\mathrm{a} Q 1}$ : June 25, 2017-September 30, 2017. Q2: October 1, 2017-December 30, 2017. Q3: December 31, 2017-March 31, 2018. Q4: April 1, 2018-June 30, 2018.

statistical analysis. Hospital rating was assessed by patients in the Press Ganey survey on a scale of 0 to 10 , with 10 being the most satisfactory response. Analysis of top-box scores was also performed for all responses, because top-box scores are the preferred method of presenting satisfaction scores from the Press Ganey survey. These scores were presented as percentage of patients assigning topbox scores. Hospital ratings of 9 or 10 and survey answers of always were designated as top-box scores.

\section{Statistical Analysis}

Statistical analysis was performed using the Statistical Package for Social Sciences, Version 23 (IBM Corp, Armonk, NY). All data were analyzed by quarter. A $\chi^{2}$ test of independence was used to compare percentages and 1-way analysis of variance was used to compare means across quarters. Significance was set at $P<.05$.

\section{RESULTS}

\section{Patient Sample}

A total of 1729 patients undergoing any spine surgery by surgeons performing at least 20 surgeries per quarter and who had medication data during hospitalization available were analyzed (48.99\% women, $51.01 \% \mathrm{men})$. The patients of 15 surgeons were included. Mean age was $63.09 \pm 13.07$ years. A total of 427 patients had their operations in Quarter 1 (Q1), 439 in Q2, 453 in Q3, and 440 in Q4.

\section{Overall Opioid Usage}

Mean opioid use in total MME per patient hospitalization across the entire year was 574.46, with no significant difference between quarters $(P=$ .116 , Table 1). Mean MME per day per patient decreased between quarters, with the highest mean 91.84 in Q2 and the lowest 77.50 in Q4 (mean =
86.92 in Q1 and 84.98 in Q3, $P=.048$; Table 1). Length of stay did not significantly change (Table $1)$.

\section{Patient Opioid Usage by Physician}

From Q1 to Q4, three physicians individually had a significantly decreased mean MME per day for their patients (Table 2). Two of these physicians also had significantly decreased total MME (Table 2). Length of stay did not significantly change for patients of Physicians 1 and 2, but it decreased for patients of Physician 3 (Table 2).

\section{Patient Opioid Usage by Procedure Type}

From Q1 to Q4, MME per day decreased (from 74.78 to $52.37, P=.046)$ and total MME trended toward decrease (from 465.73 to $341.63, P=.058$ ) for discectomy cases (Table 3). Controlling for age and revision procedures, total MME decreased for discectomies $(P=.006)$ and also trended toward a decrease for fusions $(P=.064)$.

\section{Nonopioid Pain Medication Usage}

Among all patients, acetaminophen, NSAID, and steroid prescription rates increased over time $(P=$ .001; Table 4). This was also the case in fusion patients specifically $(P=.002$; Table 4$)$. NSAID prescription rates also increased in laminectomy patients $(3.23 \%$ to $4.89 \%, P=.041)$. Concurrently, benzodiazepine and GABA analog prescriptions decreased among all patients (from $19.20 \%$ to $10.68 \%, P<.001$, and from $9.84 \%$ to $4.77 \%, P=$ .025 , respectively; Table 4). Benzodiazepine prescriptions also decreased in fusion patients specifically (from $24.51 \%$ to $12.23 \%, P<.001$ ).

\section{Press Ganey Satisfaction Survey Results}

A total of 198 patients responded to the Press Ganey Satisfaction Survey. There were no significant differences between quarters for mean hospital 
Table 2. Opioid medication usage during hospitalization per patient by quarter by individual physician for physicians that had significant changes in morphine milligram equivalents (MME) across quarters $(\mathrm{Q})^{\mathrm{a}}{ }^{\mathrm{a}}$

\begin{tabular}{|c|c|c|c|c|c|}
\hline Parameter & Q1 & Q2 & Q3 & Q4 & $P$ Value \\
\hline \multicolumn{6}{|l|}{ Physician 1} \\
\hline No. patients & 20 & 29 & 38 & 28 & \\
\hline Mean MME/d & 115.70 & 60.76 & 80.79 & 60.13 & .013 \\
\hline $95 \%$ CI & $65.60-165.79$ & $44.11-77.40$ & $61.83-99.74$ & $42.27-77.98$ & \\
\hline Mean total MME & 815.88 & 357.11 & 519.45 & 392.68 & .004 \\
\hline $95 \% \mathrm{CI}$ & $415.42-1216.33$ & $248.10-466.13$ & $393.35-645.54$ & $298.40-486.96$ & \\
\hline Length of stay, d & 6.56 & 7.60 & 7.08 & 6.12 & .168 \\
\hline \multicolumn{6}{|l|}{ Physician 2} \\
\hline No. patients & 42 & 37 & 43 & 51 & \\
\hline Mean MME/d & 92.89 & 74.51 & 66.40 & 69.53 & .042 \\
\hline $95 \%$ CI & $74.08-111.71$ & $60.26-88.77$ & $55.32-77.48$ & $57.17-82.89$ & \\
\hline Mean total MME & 706.79 & 539.39 & 484.47 & 451.72 & .014 \\
\hline $95 \% \mathrm{CI}$ & $535.02-878.55$ & $421.65-657.14$ & $382.95-585.98$ & $365.96-537.47$ & \\
\hline Length of stay, d & 8.04 & 7.04 & 8.05 & 7.87 & .485 \\
\hline \multicolumn{6}{|l|}{ Physician 3} \\
\hline No. patients & 45 & 55 & 54 & 48 & \\
\hline Mean MME/d & 75.47 & 81.90 & 75.94 & 50.92 & .023 \\
\hline $95 \% \mathrm{CI}$ & $56.81-94.12$ & $69.36-94.44$ & $58.21-93.68$ & $40.61-61.22$ & \\
\hline Mean total MME & 466.72 & 490.41 & 514.12 & 376.04 & .259 \\
\hline $95 \% \mathrm{CI}$ & $357.56-575.88$ & $401.56-579.26$ & $390.36-637.88$ & $290.43-461.66$ & \\
\hline Length of stay, $d$ & 7.08 & 7.53 & 6.86 & 5.91 & .010 \\
\hline
\end{tabular}

${ }^{\mathrm{a} Q 1}$ : June 25, 2017-September 30, 2017. Q2: October 1, 2017- December 30, 2017. Q3: December 31, 2017- March 31, 2018. Q4: April 1, $2018-J u n e ~ 30,2018$.

ratings (8.66 for Q1, 8.60 for Q2, 8.36 for Q3, and 8.40 for $\mathrm{Q} 4, P=.521$; Table 5). There were also no significant differences between quarters for responses to questions from Press Ganey Satisfaction Survey regarding how often staff talk about pain $(P=.164)$, how often staff talk about pain treatment $(P=.595)$ or whether patients recommend the hospital $(P=.096)$. This was also the case for topbox ratings for the same questions $(P=.381, .837$, and .610, respectively; Table 5). There were no significant differences between quarters for responses in all other patient satisfaction questions or their top-box ratings ( $P$ value range, .359-.988).

\section{DISCUSSION}

Effective postoperative pain management ultimately amounts to the reduction of pain and improvement of functional outcomes while mini-

Table 3. Opioid medication usage during hospitalization per patient by quarter (Q) by procedure type. ${ }^{\mathrm{a}}$

\begin{tabular}{|c|c|c|c|c|c|}
\hline Parameter & Q1 & Q2 & Q3 & Q4 & $P$ Value \\
\hline \multicolumn{6}{|l|}{ Discectomy } \\
\hline No. patients & 65 & 87 & 97 & 75 & \\
\hline Mean total MME & 465.73 & 421.35 & 367.65 & 341.63 & .058 \\
\hline $95 \% \mathrm{CI}$ & $364.38-567.07$ & $361.97-480.73$ & $313.91-421.40$ & $286.55-396.71$ & \\
\hline Mean MME/d & 74.78 & 70.34 & 64.36 & 52.37 & .046 \\
\hline $95 \% \mathrm{CI}$ & $59.36-90.20$ & $57.58-83.11$ & $55.89-72.81$ & $44.30-60.44$ & \\
\hline \multicolumn{6}{|l|}{ Fusion } \\
\hline No. patients & 253 & 256 & 292 & 278 & \\
\hline Mean total MME & 721.11 & 725.00 & 664.00 & 579.30 & .214 \\
\hline $95 \% \mathrm{CI}$ & $584.48-857.73$ & $592.59-857.41$ & $585.32-742.68$ & $490.69-667.91$ & \\
\hline Mean MME/d & 96.69 & 105.33 & 94.88 & 88.71 & .185 \\
\hline $95 \% \mathrm{CI}$ & $86.19-107.19$ & $91.98-118.68$ & $85.89-103.87$ & $79.30-98.13$ & \\
\hline \multicolumn{6}{|l|}{ Laminectomy } \\
\hline No. patients & 62 & 50 & 41 & 47 & \\
\hline Mean total MME & 468.02 & 512.70 & 474.70 & 411.81 & .704 \\
\hline $95 \% \mathrm{CI}$ & $391.80-544.25$ & $333.96-691.44$ & $363.89-585.50$ & $314.64-508.97$ & \\
\hline Mean MME/d & 70.44 & 79.33 & 73.01 & 62.71 & .474 \\
\hline $95 \% \mathrm{CI}$ & $58.21-82.66$ & $63.17-95.50$ & $57.03-89.00$ & $46.99-78.44$ & \\
\hline \multicolumn{6}{|l|}{ Other } \\
\hline No. patients & 47 & 46 & 23 & 40 & \\
\hline Mean total MME & 444.73 & 412.39 & 442.59 & 394.01 & .875 \\
\hline $95 \% \mathrm{CI}$ & $348.30-541.17$ & $320.48-504.30$ & $331.36-553.81$ & $284.86-503.17$ & \\
\hline Mean MME/d & 72.88 & 71.03 & 67.60 & 64.06 & .798 \\
\hline $95 \% \mathrm{CI}$ & $60.56-85.20$ & $60.09-81.96$ & $50.20-85.00$ & $47.26-80.86$ & \\
\hline
\end{tabular}

Abbreviations: MME, morphine milligram equivalents.

${ }^{a}$ Q1: June 25, 2017-September 30, 2017. Q2: October 1, 2017-December 30, 2017. Q3: December 31, 2017-March 31, 2018. Q4: April 1, 2018-June 30, 2018. 
Table 4. Non-opioid pain medication prescription rates during hospitalization by quarter $(\mathrm{Q}){ }^{\mathrm{a}}$

\begin{tabular}{|c|c|c|c|c|c|}
\hline Pain Medication Prescription & Q1, \% $(n=427)$ & Q2, \% $(n=439)$ & Q3, \% $(n=453)$ & Q4, \% $(n=440)$ & $P$ Value \\
\hline Acetaminophen & 9.13 & 11.62 & 13.91 & 17.05 & .001 \\
\hline NSAIDs & 6.32 & 6.38 & 7.73 & 9.77 & .048 \\
\hline Steroids & 9.13 & 11.62 & 13.91 & 17.05 & .001 \\
\hline Benzodiazepine & 19.20 & 17.08 & 13.47 & 10.68 & $<.001$ \\
\hline GABA analogs & 9.84 & 7.52 & 7.51 & 4.77 & .025 \\
\hline
\end{tabular}

Abbreviations: GABA, $\gamma$-aminobutyric acid; NSAIDs, nonsteroidal anti-inflammatory drugs.

${ }^{a}$ Q1: June 25, 2017-September 30, 2017. Q2: October 1, 2017-December 30, 2017. Q3: December 31, 2017-March 31, 2018. Q4: April 1, 2018-June 30, 2018.

mizing adverse events associated with analgesic use. Whereas opioid medications are still the standard therapy used in treating postoperative pain, growing concern about opioid overdoses in the United States has spurred institutional attempts to reduce their use and misuse in the inpatient setting. ${ }^{23,24}$ This is especially relevant in spine surgery, because these procedures are associated with high levels of postoperative pain, and existing research ${ }^{25-27}$ has shown an increased risk of inappropriate opioid prescriptions and chronic opioid use in orthopedic surgery patients. As a way to combat this, multimodal approaches to pain management relying on synergistic effects between multiple nonopioid medications have increased in popularity. At the same time, patient satisfaction is an important metric to consider when evaluating the efficacy of pain management in patient care. Thus, the purpose of this study was to examine rates of pain medication prescription and concurrent patient satisfaction in spine surgery patients at our institu- tion, where an active effort has been made to reduce opioid usage.

In the entire patient population analyzed in our study, mean opioid use in total MMEs per patient hospitalization through the entire year was 574.46. Although the mean total MME per hospitalization decreased each quarter from 615.06 in Q1 to 504.05 in Q4, there was no statistical significance. However, the mean MME per day per patient did significantly decrease between quarters, with the highest mean of 91.84 in Q2 and lowest 77.50 in Q4. Though the total amount of opioid usage per hospitalization did not decrease significantly over the course of the year, the daily amount used per patient was successfully reduced. Despite the reduction of mean MME per day, length of stay did not significantly change across quarters. This is perhaps explained by a nonsignificant trend toward decreasing total MME by quarter (615.06 in Q1 and 504.05 in Q4) that emerged upon assessing changes in usage on a day-to-day level. This suggests that the physicians at

Table 5. Patient satisfaction responses and percentage of top-box scores by quarter $(Q){ }^{\text {a }}$

\begin{tabular}{|c|c|c|c|c|c|}
\hline Hospital Rating (0 to 10) & Q1 & Q2 & Q3 & Q4 & $P$ Value \\
\hline No. patients & 59 & 35 & 58 & 43 & \\
\hline Mean $\pm \mathrm{SD}$ & $8.66 \pm 0.92$ & $8.60 \pm 0.78$ & $8.36 \pm 1.44$ & $8.40 \pm 1.76$ & .521 \\
\hline$\%$ with Top-Box Score & 83.05 & 74.29 & 75.86 & 79.07 & .720 \\
\hline \multicolumn{6}{|c|}{ How often staff talk about pain } \\
\hline No. patients & 55 & 35 & 55 & 41 & \\
\hline Never & 0 & 0 & 0 & 0 & .164 \\
\hline Sometimes & 6 & 2 & 3 & 2 & \\
\hline Usually & 13 & 13 & 12 & 6 & \\
\hline Always & 36 & 20 & 40 & 33 & \\
\hline$\%$ with Top-Box Score & 65.45 & 57.14 & 72.73 & 80.49 & .381 \\
\hline \multicolumn{6}{|c|}{ Staff talk about pain treatment } \\
\hline No. patients & 54 & 35 & 55 & 41 & \\
\hline Never & 0 & 0 & 1 & 1 & .595 \\
\hline Sometimes & 2 & 0 & 1 & 2 & \\
\hline Usually & 8 & 7 & 17 & 12 & \\
\hline Always & 44 & 28 & 36 & 26 & \\
\hline$\%$ with Top-Box Score & 81.48 & 80.00 & 65.45 & 63.41 & .837 \\
\hline \multicolumn{6}{|l|}{ Recommend the hospital } \\
\hline No. patients & 57 & 35 & 59 & 42 & \\
\hline Never & 1 & 0 & 1 & 1 & .096 \\
\hline Sometimes & 3 & 0 & 1 & 1 & \\
\hline Usually & 7 & 4 & 9 & 3 & \\
\hline Always & 46 & 31 & 48 & 37 & \\
\hline$\%$ with Top-Box Score & 80.70 & 88.57 & 81.36 & 88.10 & .610 \\
\hline
\end{tabular}

${ }^{a}$ Q1: June 25, 2017-September 30, 2017. Q2: October 1, 2017-December 30, 2017. Q3: December 31, 2017-March 31, 2018. Q4: April 1, 2018-June 30, 2018. Hospital rating of 9 or 10 and answer choice always are considered top-box score responses. 
our institute overall were successful in reducing opioid usage as the year progressed.

Subanalyzing patients by physician, we found that 3 physicians had decreasing mean MME per day values for their patients. Two of these physicians also had decreased total MME. None of the other physicians had statistically significant decreases in total MME or MME per day throughout the year, though most did demonstrate lower mean values in Q4 compared with Q1. This is likely attributable to smaller sample sizes per quarter and highlights the benefit of examining an aggregate of cases. When we assessed opioid usage by procedure type, MME per day decreased (from 74.78 to 52.37) and total MME trended toward a significant decrease (from 465.73 to 341.63 ) for discectomy cases. After controlling for age and revision, we found that total MME decreased for discectomies and also trended toward a decrease for fusions.

Along with decreased opioid use, there was a concurrent increase in prescriptions for other, nonopioid pain medications during the year. Among all patients, prescription rates for acetaminophen, NSAIDs, and steroids all increased throughout the year. NSAID prescription rates also increased in laminectomy patients. These changes highlight an increasing use of nonopioid medications to manage postoperative pain at our institution. At the same time, benzodiazepine and GABA analog prescriptions decreased among all patients over the course of the year. Benzodiazepine prescriptions also decreased in fusion patients specifically. Side effects of benzodiazepines and GABA analogs can be synergistic with the inhibitory effects from opioids due to action on the same receptors, so a concomitant decrease in their usage in pain regimens is a promising development. ${ }^{28}$ In particular, the combination of opioid medications with benzodiazepines increases the risk of respiratory depression and death, and a 2016 study by Dasgupta et al found a nearly 10 -fold increase in risk of death from overdose in outpatients concurrently using highdose opioids and benzodiazepines. ${ }^{29,30}$ Therefore, decreases in benzodiazepine and GABA analog prescriptions for postoperative patients already on opioids should also improve safety.

In spite of changes in medication use, there were no cumulative differences in patient satisfaction throughout the year. Mean hospital ratings from the Press Ganey Satisfaction Survey did not change significantly across quarters (8.66 for Q1, 8.60 for
Q2, 8.36 for Q3, and 8.40 for Q4). There were also no differences between quarters in responses to questions regarding how often hospital staff talk about pain, how often staff talk about pain treatment, or whether the patient would recommend the hospital. This was also the case for top-box ratings for the same questions, with the majority of patients selecting the top-box response for each question in each quarter. Altogether, this establishes that, over the studied time period, opioid use decreased and nonopioid prescriptions increased during hospitalization, whereas patient satisfaction scores remained unchanged.

Our study has some limitations. Because this was a retrospective cohort study, it has all of the accompanying limitations of such a study, such as the potential introduction of selection bias and information bias. We also had limited sample sizes when splitting patients up by physician, which may have limited our ability to detect the significance of changes in the use of both opioid and nonopioid medications throughout the year. With Press Ganey survey data available for significantly fewer patients than medication use data, we may not be seeing fully the potential changes in patient satisfaction over the year. Recent changes in Press Ganey survey questions about pain management may have also affected our satisfaction data. Some questions in the survey were changed in 2017 , during our study time frame, to reflect a greater emphasis on communication between providers and patients about pain rather than patient perception about how successful pain treatment was. The question about how often hospital staff talked about pain replaced a similar question about how often pain was "well controlled." Similarly, the question about how often staff talked about pain treatment replaced one about whether the staff "did everything they could" to help with pain. Although these replacement questions were thematically related, their different focus may have led patients to answer differently than in the original questions.

Future studies could benefit from implementing a lower opioid-use protocol when examining opioid prescriptions and patient satisfaction scores. As more institutions attempt to reduce their opioid usage, findings in these projects could also be weighed against the possibility of underprescribing for pain. In addition, certain procedure types had more significant differences in drug use at our institution, and further exploration into what 
accounts for these differences may be beneficial. For example, postoperative opioid usage tended to be higher overall in fusion cases but reductions in use were more significant in discectomy cases. This may be related to the invasiveness of these procedures. Because the more invasive fusions likely necessitated more opioid usage for pain control, they may have been less liable to significant opioid reductions. Meanwhile, the less invasive discectomies may have proven to require minimal opioid usage in the first place, without further reductions negatively affecting pain. This also highlights that further adjustments in pain regimens could still be made, depending on procedure type. Overall, the findings of our study indicate an increasing effort in reducing opioid use among providers and suggest the ability to do so without impacting overall satisfaction rates.

\section{CONCLUSIONS}

Over the studied time period, opioid use decreased and nonopioid prescriptions increased during hospitalization, whereas satisfaction scores remained unchanged. These findings indicate an increasing effort in reducing opioid use among providers and suggest the ability to do so without impacting overall satisfaction rates.

\section{REFERENCES}

1. Phillips FM, Slosar PJ, Youssef JA, Andersson G, Papatheofanis F. Lumbar spine fusion for chronic low back pain due to degenerative disc disease: a systematic review. Spine (Phila Pa 1976). 2013;38(7). doi:10.1097/BRS. 0b013e3182877f11

2. Dunn LK, Durieux ME, Fernández LG, et al. Influence of catastrophizing, anxiety, and depression on in-hospital opioid consumption, pain, and quality of recovery after adult spine surgery. J Neurosurg Spine. 2017;28(1):119-126. doi:10.3171/ 2017.5.spine 1734

3. Gottschalk A, Durieux ME, Nemergut EC. Intraoperative methadone improves postoperative pain control in patients undergoing complex spine surgery. Anesth Analg. 2011;112(1):218-223. doi:10.1213/ANE.0b013e3181d8a095

4. Carli F, Kehlet H, Baldini G, et al. Evidence basis for regional anesthesia in multidisciplinary fast-track surgical care pathways. Reg Anesth Pain Med. 2011;36(1):63-72. doi:10.1097/ AAP.0b013e31820307f7

5. Boezaart AP, Davis G, Le-Wendling L. Recovery after orthopedic surgery: techniques to increase duration of pain control. Curr Opin Anaesthesiol. 2012;25(6):665-672. doi:10. 1097/ACO.0b013e328359ab5a

6. Armaghani SJ, Lee DS, Bible JE, et al. Increased preoperative narcotic use and its association with postoperative complications and length of hospital stay in patients undergo- ing spine surgery. J Spinal Disord Tech. 2014;(615). doi:10.1097/ BSD.0000000000000109

7. Pugely AJ, Martin CT, Gao Y, Mendoza-Lattes S. Causes and risk factors for 30-day unplanned readmissions after lumbar spine surgery. Spine (Phila Pa 1976). 2014;39(9):761768. doi:10.1097/BRS.0000000000000270

8. Cantrill SV, Brown MD, Carlisle RJ, et al. Clinical policy: critical issues in the prescribing of opioids for adult patients in the emergency department. Ann Emerg Med. 2012;60(4):499525. doi:10.1016/j.annemergmed.2012.06.013

9. Inturrisi CE. Clinical pharmacology of opioids for pain. Clin J Pain. 20020;18(suppl 4):S3-S13. doi:10.1097/00002508200207001-00002

10. Devin CJ, McGirt MJ. Best evidence in multimodal pain management in spine surgery and means of assessing postoperative pain and functional outcomes. $J$ Clin Neurosci. 2015;22(6):930-938. doi:10.1016/j.jocn.2015.01.003

11. Cauley CE, Anderson G, Haynes AB, Menendez M, Bateman BT, Ladha K. Predictors of in-hospital postoperative opioid overdose after major elective operations: a nationally representative cohort study. Ann Surg. 2017;265(4):702-708. doi:10.1097/SLA.0000000000001945

12. Boscarino JA, Rukstalis M, Hoffman SN, et al. Risk factors for drug dependence among out-patients on opioid therapy in a large US health-care system. Addiction. 2010;105(10):1776-1782. doi:10.1111/j.1360-0443.2010.03052.x

13. Warner M, Chen LH, Makuc DM, Anderson RN, Miniño AM. Drug poisoning deaths in the United States, 1980 2008. NCHS Data Brief. 2011;(81):1-8. PMID: 22617462.

14. Kamdar MM. Principles of analgesic use in the treatment of acute pain and cancer pain, sixth edition. $J$ Palliat Med. 2010;13(2):217-218. doi:10.1089/jpm.2010.9854

15. Garcia RM, Cassinelli EH, Messerschmitt PJ, Furey CG, Bohlman HH. A multimodal approach for postoperative pain management after lumbar decompression surgery. J Spinal Disord Tech. 2012;26(6):291-297. doi:10.1097/bsd. 0b013e318246b0a6

16. Sinatra RS, Torres J, Bustos AM. Pain management after major orthopaedic surgery: current strategies and new concepts. J Am Acad Orthop Surg. 2002;10(2):117-129. doi:10. 5435/00124635-200203000-00007

17. Kurd MF, Kreitz T, Schroeder G, Vaccaro AR. The role of multimodal analgesia in spine surgery. $\mathrm{J}$ Am Acad Orthop Surg. 2017;25(4):260-268. doi:10.5435/JAAOS-D-16-00049

18. Soroceanu A, Ching A, Abdu W, McGuire K. Relationship between preoperative expectations, satisfaction, and functional outcomes in patients undergoing lumbar and cervical spine surgery: a multicenter study. Spine (Phila $\mathrm{Pa}$ 1976). 2012;37(2):E103-E108. doi:10.1097/BRS 0b013e3182245c1f

19. Yamashita K, Hayashi J, Ohzono K, Hiroshima K. Correlation of patient satisfaction with symptom severity and walking ability after surgical treatment for degenerative lumbar spinal stenosis. Spine (Phila Pa 1976). 2003;28(21):2477-2481. doi:10.1097/01.BRS.0000090888.63860.4F

20. Rogers F, Horst M, To T, et al. Factors associated with patient satisfaction scores for physician care in trauma patients. J Trauma Acute Care Surg. 2013;75(1):110-115. doi:10.1097/ TA.0b013e318298484f

21. Zgierska A, Miller M, Rabago D. Patient satisfaction, 
prescription drug abuse, and potential unintended consequences. JAMA. 2012;307(13):1377-1378. doi:10.1001/jama.2012.419

22. Jirarattanaphochai K, Thienthong S, Sriraj W, et al. Effect of parecoxib on postoperative pain after lumbar spine surgery: a bicenter, randomized, double-blinded, placebocontrolled trial. Spine (Phila Pa 1976). 2008;33(2):132-139. doi:10.1097/BRS.0b013e3181604529

23. CDC grand rounds: prescription drug overdoses - a U.S. epidemic. MMWR Morb Mortal Wkly Rep. 2012;61(1):10-13. PMID: 22237030. http://proxy.cc.uic.edu/login?ur=http://search. ebscohost.com/login.aspx?direct $=$ true $\& \mathrm{db}=\mathrm{rzh} \& \mathrm{AN}=104632195$.

24. Lovecchio F, Premkumar A, Stepan JG, Albert TJ. Fighting back: institutional strategies to combat the opioid epidemic: a systematic review. HSS J. 2019;15(1):66-71. doi:10. 1007/s11420-018-09662-y

25. Jiang X, Orton M, Feng R, et al. Chronic opioid usage in surgical patients in a large academic center. Ann Surg. 2017;265(4):722-727. doi:10.1097/SLA.0000000000001780

26. Helmerhorst GTT, Vranceanu AM, Vrahas M, Smith M, Ring D. Risk factors for continued opioid use one to two months after surgery for musculoskeletal trauma. J Bone Joint Surg Am. 2014;96(6):495-499. doi:10.2106/JBJS.L.01406

27. Schoenfeld AJ, Harris MB, Liu H, Birkmeyer JD. Variations in Medicare payments for episodes of spine surgery. Spine J. 2014;14(12):2793-2798. doi:10.1016/j.spinee.2014.07. 002

28. Cunningham JL, Craner JR, Evans MM, Hooten WM. Benzodiazepine use in patients with chronic pain in an interdisciplinary pain rehabilitation program. J Pain Res. 2017;10:311-317. doi:10.2147/JPR.S123487
29. Hirschtritt ME, Delucchi KL, Olfson M. Outpatient, combined use of opioid and benzodiazepine medications in the United States, 1993-2014. Prev Med Reports. 2018;9:49-54. doi:10.1016/j.pmedr.2017.12.010

30. Dasgupta N, Funk MJ, Proescholdbell S, Hirsch A, Ribisl KM, Marshall S. Cohort study of the impact of highdose opioid analgesics on overdose mortality. Pain Med (United States). 2016;17(1):85-98. doi:10.1111/pme.12907

Disclosures and COI: The authors received no funding for this study and report no conflicts of interest.

Corresponding Author: Charla R. Fischer, MD, NYU Medical Center-NYU Langone Orthopedic Hospital, Department of Orthopedic Surgery, 145 East $32^{\text {nd }}$ Street, $4^{\text {th }}$ Floor, New York, NY 10016. Phone: (646) 356-9415; Fax: (646) 356-9413; Email: Charla.Fischer@nyulangone.org.

Published 22 January 2021

This manuscript is generously published free of charge by ISASS, the International Society for the Advancement of Spine Surgery. Copyright (C) 2020 ISASS. To see more or order reprints or permissions, see http://ijssurgery.com. 